\title{
Checklist of plant species of the coastal fynbos and rocky head- lands, south of George, South Africa
}

\author{
J.E. VICTOR**, D.B. HOARE*' and R.A. LUBKE*
}

Keywords: checklist, coastal fynbos, endemics, phytogeography, rocky headlands, South Africa. Western Cape

\section{ABSTRACT}

\begin{abstract}
A checklist of vascular plants and cryptograms was compiled for the fynbos and rocky headland communities of the coastal region south of George. The area studied is a $12 \mathrm{~km}$ stretch of steep sandstone cliffs forming alternating bays and headlands situated between Glentana and Wilderness. The plant communities of the natural vegetation inhabiting the coastline are a mixture of coastal thicket, riparian thicket, fynbos and rocky headland types. The extent of natural vegetation has heen reduced by the spread of agricultural land and urban development and is under further threat from the spread of naturalised alien invader species, particularly Acacia cyclops. The checklist records the occurrence of 271 taxa including 16 alien species ( $6 \%$ of taxa). Of the flowering plant species recorded, $6 \%$ were regional or local endernics.
\end{abstract}

\section{INTRODUCTION}

The study area is a $12 \mathrm{~km}$ section of coastline south of George extending from Rooiklip, southeast of Pacaltsdorp, to Ghwanobaai, $3 \mathrm{~km}$ east of Glentana (see Hoare et al. 2000 for details). It includes a band of vegetation within $500 \mathrm{~m}$ of the high tide mark on steep sandstone cliffs which form alternating bays and headlands. The plant communities of the natural vegetation along the coastline are a mixture of coastal thicket, riparian thicket, fynbos and rocky headland types. The study was confined to the fynbos and rocky headland vegetation. Riparian thicket, dune thicket, dense alien stands and agricultural lands were not sampled. Rainfall along this section of coastline occurs throughout the year, but with three distinct peaks in spring, summer and autumn (Hoare et al. 2000). Because of its close proximity to the sea, vegetation structure and composition are greatly influenced by oceanic winds. The extent of the natural vegetation has been reduced by the spread of agricultural land and urban activities and is under further threat from the spread of naturalised alien species, particularly Acacia cyclops.

This study area falls into that part of the Fynbos Biome called Limestone Fynbos of the Mossel Bay District (Low \& Rebelo 1996) of which $\pm 14 \%$ is conserved and $40 \%$ transformed, although the recent proclamation of the Agulhas National Park may affect these statistics. The particular study area is of interest because of its scenic beauty and its location in the centre of the Garden Route- a popular tourist attraction. Most of the study area is owned by private landowners who have used the land mostly for agriculture (in places agricultural lands extend to within a few metres of the summit of the coastal cliffs), but also for plantation forestry on a

\footnotetext{
* National Botanical Institute. Private Bag X101, 0001 Pretoria

* Department of Botany. Rhodes University. 6140 Grahamstown. South Africa.

† Present address. Agricultural Research Council. Range \& Forage Institute, Private Bag X05, (0)39 Lynn East, Pretoria

MS received: 1999-11-29.
}

small scale. Urban development occurs in nodes, e.g. Herold's Bay and Victoria Bay. Recreational activities, e.g. fishing, occur on a small scale along the coast. Disturbances related to all these activities have resulted in invasion by alien trees and shrubs, particularly Acacia cyclops. Pinus species have spread from the plantations and are also a potentially serious threat.

The aim of this work was to provide a checklist of the plant species along this stretch of coastline that can act as a basic reference for floristic and ecological work and as a baseline for future development in the region. This checklist forms a link between checklists and floristic studies done for the Western Cape coastal region (Boucher 1977; Taylor 1985), southern Cape coast (Hellström 1990; Hoare 1994; Van der Merwe 1979) and the Eastern Cape coast (Lubke 1983; Lubke et al. 1988; Cloete \& Lubke 1999). This provides the opportunity for comprehensive examination of floristic gradients along the coastal region of the Fynbos Biome.

\section{METHODS}

Fieldwork was done in all four seasons of the year to cover as many flowering times as possible. Voucher specimens of most taxa were deposited in the National Herbarium, Pretoria, and additional taxa listed were obtained from sight records made during the course of fieldwork. The herbarium collection at PRE was consulted to obtain records of plant species previously collected in the study area, and these were added to the checklist.

\section{RESULTS}

The checklist lists 271 taxa comprising nine lichen species, three bryophytes, three pteridophytes, two gymnosperms, 56 monocotyledons and 198 dicotyledons (Table 1). The most commonly represented angiosperm families (Table 2) are Asteraceae (15\% of species), Poaceae (7\%), Cyperaceae $(6 \%)$, Fabaceae $(6 \%)$, Mesembryanthemaceae $(5 \%)$, Ericaceae $(5 \%)$ and Crassulaceae $(4 \%)$. The genera with the most species are Erica (11), Crassula (11), Aspalathus (8) and Helichrysum (6). Ficinia, Lam- 
TABLE 1.-Number of families, genera and species recorded in the vegetation of the coast south of George

\begin{tabular}{lcccc}
\hline & Families & Genera & $\begin{array}{c}\text { Indigenous } \\
\text { spp. }\end{array}$ & $\begin{array}{c}\text { Naturalised } \\
\text { alien spp. }\end{array}$ \\
\hline Lichens & & 4 & 9 & 0 \\
Bryophytes & 3 & 3 & 3 & 0 \\
Pteridophytes & 3 & 3 & 3 & 0 \\
Gymnosperms & 1 & 1 & 0 & 2 \\
Angiosperms & & & & 11 \\
$\quad$ Dicotyledons & 52 & 107 & 187 & 3 \\
$\quad$ Monocotyledons & 10 & 41 & 53 & 16 \\
Total & 69 & 159 & 255 & \\
\hline
\end{tabular}

pranthus, Phylica and Hermannia were each represented by five species.

All of the 20 largest genera listed for the Cape flora by Bond \& Goldblatt (1984) are represented in this coastal area, as are 13 of the 15 largest families. Of the 16 alien species recorded, Acacia cyclops was by far the most abundant.

Some 230 flowering plant species were classified according to phytogeographical range and affinity (Table 3 ). It was found that $35 \%$ are endemic to the Fynbos Biome and $7 \%$ are regional (southern Cape) endemics and one was a local endemic--Silene vlokii, which has a restricted range from Herold's Bay to Glentana.

\section{DISCUSSION}

Asteraceous Coastal Fynbos is defined as having high asteraceous and non-ericaceous ericoid cover and often high grass cover (Cowling 1992). Phylica, Passerina, Agathosma (and other Diosminae), Aspalathus, Restio and Cliffortia are listed as dominant genera in this vegetation type (Cowling 1992), a view which is consistent with what was found in the study area (Hoare et al. 2000).

A comparison of the flora of the study area with those of the Goukamma Nature Reserve (Table 4) shows that the number of species and genera in the present study area is comparatively high in relation to its size, especially considering that not all vegetation types were sampled. Goukamma Nature Reserve is a larger area but with

TABLE 2.-Families of angiosperms in the study with the highest number of genera and species

\begin{tabular}{lcc}
\hline Family & No. genera & No. spp. \\
\hline Asteraceae & 25 & 39 \\
Poaceae & 12 & 17 \\
Cyperaceac & 8 & 16 \\
Fabaceae & 7 & 16 \\
Mesembryanthemaceae & 7 & 14 \\
Ericaceae & 3 & 13 \\
Crassulaceae & 2 & 11 \\
Iridaceae & 8 & 9 \\
Santalaceac & 3 & 7 \\
Thymelaeaceide & 2 & 5 \\
Sterculiaceac & 1 & 5 \\
\hline
\end{tabular}

TABLE 3.-Distribution of angiosperms found in the study area

\begin{tabular}{lc}
\hline Distribution & No. spp. \\
\hline Endemic to Fynbos Biome & 81 \\
Regional endemics (southern Cape) & 15 \\
Local endemics & 1 \\
\hline
\end{tabular}

TABLE 4.-Comparison of the indigenous angiosperm flora of the study area with nearby Goukamma Nature Reserve (Van der Merwe 1979; Hoare 1994)

\begin{tabular}{lcc}
\hline & Study area & $\begin{array}{c}\text { Goukamma } \\
\text { Nature } \\
\text { Reserve }\end{array}$ \\
\hline Area $\left(\mathrm{km}^{2}\right)$ & 1.9 & 14.6 \\
No. genera & 136 & 121 \\
No. spp. & 2.54 & 168 \\
No. spp. per genus & 1.7 & 1.4 \\
No. spp. per km ${ }^{2}$ & 1.34 & 11.5 \\
\hline
\end{tabular}

fewer species, indicating that there is lower diversity in the Dune Fynbos and thicket vegetation of that region compared with the communities along the rocky shore and promontories of this study area. This coastal region therefore has a surprisingly high diversity for such a small area, probably due to its abundance of microhabitats. Further studies encompassing the non-fynbos vegetation types should be carried out to contribute to the knowledge of this relatively understudied and poorly conserved region.

\section{REFERENCES}

BOND, P \& GOLDBLATT, P 1984. Plants of the Cape Flora a descriptive catalogue. Journal of South African Botany Suppl. Vol. 13: 455

BOUCHER, C. 1977. A provisional checklist of flowering plants and ferns in the Cape Hangklip area Journal of South African Botany 43: 57-80

BRUMMITT, R.K \& POWELL. C.E. 1992 . Authors of plant names. Royal Botanic Gardens, Kew, London

CLOETE, E.C. \& LUBKE. R.A. 1999. Flora of the Kap River Reserve, Eastern Cape, South Africa. Bothalia 29: 139-149

COWLING, R.M 1992. The ecology of funbos: nutrients, fire and diversity. Oxford University Press, Cape Town

HELLSTROM. G. 1990. A phytosociolengical classification, with mamagement proposals, of the Robberg Nature Reserve, Plettenbers Bay: M.Sc. thesis, Rhodes University, Grahamstown.

HOARE, D.B. 1994. Assessing successional effects on plant diversity in the Goukamma Nature Reserve, southern Cape. B.Sc. (Hons) thesis, Rhodes University, Grahamstown.

HOARE, D.B. VICTOR, JE., LUBKE, R.A \& MUCINA, L 2()(K) Vegetation of the coastal fynbos and rocky headlands south of George, South Africa. Bothalia 30): 87-96.

LOW, A.B \& REBELO, A.G. 1996. Vegelation of South Africa. Lesotho and Swaziland. Department of Environmental Afratrs \& Tourism, Pretoria.

LUBKE, R.A. 1983. A survey of the coastal vegetation near Por Alfred. eastern Cape. Botholio 14: 725-738

LUBKE, R.A., GESS, FW \& BRUTON. MN 1988 . A ficld suide w the eastern Cape coust. Wildlife Society. Grahamstown.

TAYLOR. HC. 1985. An analysis of the flowering plants and terns of the Cape of Good Hope Nature Reserve South African Journal of Boluny $51: 1-13$.

VAN DER MERWE C V 1979. Plantekologiese uspekte en bestuursprobleme van die Goukamma-natuarresenaat. MSc. thesis, University of Pretoria. 


\section{CHECKLIST}

Taxa are arranged alphabetically, and author citations follow Brummitt \& Powell (1992). Except for site records, collectors' names and numbers follow the author citation; specimens are housed at PRE. Naturalised alien species are marked with an asterisk*. Abbreviations for collectors' names: $B o$, P. Bohnen; $D a$, G. Davidse; Th, M.F. Thompson; V, J.E. Victor; VFC, C.M. Van Wyk, A. Fellingham \& M. O’Callaghan; V\&H, J.E. Victor \& D.B. Hoare; Wi, I.J.M. Williams.

\section{LICHENS}

Cladia aggregata ( $S w$.$) Nyl., V 327$

Cladonia

Chasmariae sp., $V 328$

chlorphaea (Floerke) Spreng., V 325

Cocciferae sp., V 326

confusa R.Sant., V 324

coniocraea (Floerke) Spreng., V $328 b$

Pycnoporus sp., V 545

Teloschistes flavicans ( $S$ w.) Norm., V 546

Usnea rubicunda Stirt.. V 547

\section{BRYOPHYTES}

\section{FUNARIACEAE}

Funaria hygrometrica Hedw., V 290

PHYLLOGONIACEAE

Catagonium nitens (Brid.) Card. subsp. maritimum (Hook.) S-H.Lin, V 352 POTTIACEAE

Tortella xanthocarpa (C.Muell.) Broth., V 303

\section{PTERIDOPHYTES}

\section{ADIANTACEAE}

Cheilanthes hirta Sw. var. hirta, V 367

ASPLENIACEAE

Asplenium rutifolium (P.J.Bergius) Kunze, V 351, 366

SCHIZAEACEAE

Schizaea pectinata (L.) Sw., V 317

\section{GYMNOSPERMS}

\section{PINACEAE}

Pinus

*pinaster Aiton

"radiata D.Don

\section{ANGIOSPERMS: MONOCOTYLEDONS}

\section{ASPHODELACEAE}

Anthericum cooperi Baker, V 561; V\&H 43

\section{CYPER ACEAE}

Ficinia

albicans Nees, $V \& H 83$

of gracilis (Poir.) Schrad., V 259,321,329, 359

laciniata (Thunb.) Nees, Da 33719

nigrescens (Schrad.) J Raynal, V 261.285; V\&H 68

repens (Nees) Kunth, $V 292$

Fuirena hirsuta (P.J.Bergius) P.L.Furbes, V 306

Isolepis tenuissima (Nees) Kunth, $V 288$

Mariscus

congestus (Vahl) C.B.Clarke, V 557

thunhergii (Vahl) Schrad., V 282

Pycreus polystachyos (Rotth.) Beauv. var. polystachyos, V 287

Schoenoxiphium sparteum (Wahlenb.) C.B.Clarke. Da 33726

Tetraria

bolusii C.B.Clurke, VFC 2.34

compressa Turrill, $V 246 ; V \& H 40$

Cuspidata (Romb.) C.B.Clarke. V 242, V\&H 78

microstachys (Vahl) Pfeiffer, V 309, 316, 333

Trianoptiles capensis (Sicud.) Hari., V 289

\section{HYACINTHACEAE}

Lachenalia bulbifera (Cyr.) Engl., VFC 170

Ornithogalum sp.

HYPOXIDACEAE

Einpodium sp., V 207

Spiloxene trifurcillata (Nel) Fourc, V 335

\section{IRIDACEAE}

Babiana fourcadei G.J.Lewis, V 349

Bobartia aphylla (L.f.) Ker Gawl., V 307, 540; V\&H 47

Chasmanthe aethiopica (L.) N.E.Br., VFC 171

Freesia

alba (G.L.Mey.) Gumbleton

leichtlinii Klatt. V 296

Gladiolus floribundus Jacy. subsp. floribundus, Th 609

Hesperantha falcata (L.f.) Ker Gawl., V 213

Micranthus alopecuroides (L.) Rothm.. V 558

Tritoniopsis antholyza (Poir.) Goldblatt. V 495

JUNCACEAE

Juncus

acutus $L$. subsp. leopoldii (Parl.) Snog.. V 345

dregeanus Kunsh, V 286, 534

\section{ORCHIDACEAE}

Disperis capensis (L.) SW: var. capensis, $V 238$

Herschelianthe hians (L.f.) Rauscher, V 529, 533

\section{POACEAE}

Cynodon dactylon (L.) Pers., V 516

Eragrostis

capensis (Thunb.) Trin., V 503

plana Nees, V $5 I I$

Ehrharta

calycina J.E.Sm.. V 513

capensis Thunb., V 513b: V\&H 80

erecta Lam. var. erecta

*Lolium perenne L., V 524

Paspalum

*dilatatum Poir., V 522

distichum $L ., V 517$

Pentaschistis eniostoma (Nees) Stapf

Polypogon strictus Nees, V 350

Setaria sphacelata (Schum.) Moss var. sphacelata. V 523

Sporobolus

africanus (Poir.) Robyns \& Tourmav, V 518

virginicus (L.) Kunth

Stenotaphrum secundatum (Walt.) Kuntze

Themeda triandra Forssk. $V 510$

Tribolium uniolae (L.f.) Renvoize. V\&H 8I

PONTEDERIACEAE

*Eichhornia crassipes (Mart.) Solms-Lauh., V 562

RESTIONACEAE

Hypodiscus willdenowia (Nees) Mast., Da 33722

Ischyrolepis triflora (Rottb.) Linder

Restio triticeus Rottb. V 253, 254,320, V\&H 36

Thamnochortus cinereus H.P. Linder. $V 308: V \& H 45$

\section{DICOTYLEDONS}

\section{ACANTHACEAE}

Hypoestes aristata (Vuhl) Rorem. \& Schult. var. thiniorum Balkwill, V 34 I AIZOACEAE

Tetragonia

fruticosa $L ., V 273$

decumbens Mill

spicata $L . f$. var. spicata. $V 338$

virgata Schltr. V\&H 42

\section{ANACARDIACEAE}

Rhus

crenata Thumb., V 302

glauca Thunb. VFC 177

lucida $L$. forma lucida, $V 208$

APIACEAE

Centella virgata (L.f.) Drude. V 319

APOCYNACEAE

Astephanus marginatus Decne

Sarcostemma viminale (L.) R Br 


\section{ASTERACEAE}

Arctotheca prostrata (Salisb.) Britten, VFC 185

Athanasia trifurcata $(L)$.$L ., V 509$

Athrixia capensis Ker Gawl., V $54 I$

Berkheya armata (Vahl) Druce, V 531

Chrysanthemoides monilifera (L.) Norl. subsp. pisifera (L.) Norl., V 262

Cineraria britteniae Hutch. \& RA.Dyer, V\&H 39, 71

Cullumia bisulca (Thunb.) Less.

Disparago kraussii Sch. Bip., V 502; V\&H 58

Elytropappus rhinocerotis (L.f.) Less., $V 548$

Eriocephalus africanus $L ., V 244 ; V \& H 33$

Felicia

amoena (Sch.Bip.) Levyns, subsp. latifolia Grau, V 536

filifolia (Vent.) Burtt Davy, subsp. bodkinii (Compton) Grau, V 300

Gazania rigens (L.) Gaertn. var. uniflora (L.f.) Rössler, V 294; V\&H 75

Gerbera serrata (Thunb.) Druce, V 239; V\&H 66

Helichrysum

anomalum Less., $V 240,514$

asperum (Thunb.) Hilliard \& B.L.Burtt var. glabrum Hilliard, V 532

cymosum (L.) D.Don subsp. cymosum, VFC 227

felinum Less., $V 322$

odoratissimum (L.) Sweet, V 564

teretifolium (L.) D. Don, V 235

Metalasia

acuta Karis, $V 217$

pungens D.Don, $V 247 ; V \& H 52,54 b$

Oedera

capensis (L.) Druce, $V 236$

imbricata Lam., V 267

Othonna carnosa Less., var. camosa, V 370

Plecostachys serpyllifolia (Berg.) Hilliard \& B L Burtt, V 301

Printzia polifolia (L.) Hutch., V 323

Relhania

calycina (L.f.) L'Hér. subsp. calycina, V 364

pungens L'Hér. subsp. pungens, V 501

Senecio

angulatus $L . f$., $V 348$

deltoideus Less., VFC 183

ilicifolius $L ., V 508$

Stoebe

microphylla $D C, V$ 357: $V \& H 72,82$

plumosa (L.) Thunb., V 304

Syncarpha

canescens (L.) B.Nord

paniculata (L.) B.Nord

Tarchonanthus camphoratus $L . V$ 34: V\&H 44

Ursinia

heterodonta (DC.) N.E.Br., V 252, 528

saxatilis N.E.Br., V\&H 34

BRASSICACEAE

Heliophila subulata DC., V 334, 527

CAMPANULACEAE

Lightfootia

divaricata H.Buek var. debilis (Sond.) Adamson, VFC 95

fasciculata (L.f.) A.DC., V 500

CARYOPHYLLACEAE

Silene vlokii Mass., V 864

*Spergula arvensis $L ., V 2 / 4$

CELASTRACEAE

Cassine papillosa (Hochst.) Kuntze

Pterocelastrus tricuspidatus (Lam.) Sond., V 299

Putterlickia pyracantha (L.) Szyszyl., V 549

CHENOPODIACEAE

Sarcocornia natalensis (Ung.-Sternb.) A.J.Scott var. natalensis

CONVOLVULACEAE

Falkia repens $L . f$., $V 28 l$

CRASSULACEAE

Adromischus caryophyllaceus (Burm.f.) Lem., V 552; V\&H 37

Crassula

atropurpurea (Haw.) Diefr. var atropurpurea, $V \& H 38$

biplanata Haw., VFC 169

decumbens Thunb. var brachyphylla (Adamson) Tolken, $V 2 I$

lactea Soland., V 34

nudicaulis $L$. var. nudicaulis, $V 553$

orbicularis $L, V 297$

rubricaulis Eckl. \& Zeyh.. V 343

rupestris Thunh. subsp. rupestris, V 551 southii Schönland subsp. sphaerocephala Tölken, V 223

subulata $L$. var. fastigiata (Schönland) Tölken, V 382

DROSERACEAE

Drosera sp., $V 318$

\section{EBENACEAE}

Diospyros

dichrophylla (Gand.) De Winter, V 209

lycioides Desf. subsp. lycioides, V\&H 49

Euclea

crispa (Thunb.) Guerke subsp. crispa, V 368

natalensis $A . D C$. subsp. obovata $F$. White, $V \& H+1$

ERICACEAE

Blaeria ericoides $L, V 543$

Erica

canaliculata Andr., V $2 / 2$

discolor Andr. var. discolor, $V$ 233, 293

formosa Thunb., V 243, 323, 355; V\&H 25, 46

glandulosa Thunb. var. glandulosa, V 555; V\&H 48

hispidula $L$. var. hispidula, $V 258,356$

imbricata $L ., V 354$

peltata Andr., $V \& H 26$

speciosa Andr., V 241

triceps Link, V 559

versicolor Wend

$\mathrm{sp}, \mathrm{V} \& H 24$

Salaxis axillaris (Thunb.) G.Don, V 258, 530

EUPHORBIACEAE

Clutia

alaternoides $L$. var. brevifolia Sond

laxa Sond. VFC 218

Euphorbia cf. caterviflora N.E.Br., V 272

FABACEAE

Acacia

*cyclops G.Don, V 360

*mearnsii De Wild., V 257; V\&H 54a

Amphithalea fourcadei Compton, $V 358$

Aspalathus

alopecurus Benth., V 353; V\&H 56

asparagoides L.f. subsp. asparagoides, V\&H 21

asparagoides $L$.f. subsp. rubro-fusca (Eckl. \& Zeyh.) R.Dahlgren, V 33 I ciliaris $L ., V 526, V 544$

florifera R.Dahlgren, $V 248$

kougaensis (R.Dahlgren) R.Dahlgren, V 266; V\&H 22

laricifolia P.J.Bergius subsp. laricifolia, $V \& H 55$

nigra $L$.

Indigofera heterophylla Thunb, V 27I; V\&H 59, 77

*Lotus subbiflorus Lag., V 505

Rhynchosia

capensis (Burm.) Schinz, V 315

ciliata (Thunb.) Schinz

* Vicia sativa $L ., V 381,560$

FUMARIACEAE

*Fumaria muralis Koch subsp. muralis, $V 2 I I$

GENTIANACEAE

Chironia baccifera $L$., V 336

GERANIACEAE

Pelargonium

candicans Sprens, V 280

capitatum (L.) L'Hér. V\&H 74

caucalifolium Jacy. subsp. convolvulifolium (Kunth) J.J.A.van der Walt, V 538

fruticosum (Cav.) Willd, V 311

LAMIACEAE

Stachys graciliflora Presl, VFC I64

LAURACEAE

Cassytha ciliolata Nees, $V 227 ; V \& H 76$

LOBELIACEAE

Lohelia

bicolor Sim.s, V\&H 6

coronopifolia $L, V 255,537$

erinus $L$., $V 520$

torrentosa $L$.

Monopsis unidentata (Drvand) E. Wimm subsp. unidentata, V $\$ / 5$

MALVACEAE

Anisodontea scabrosa (l, ) Bates, V 279

Hibiscus acthiopicus I var ovatus Harv, V\&H 57 


\section{MESEMBRYANTHEMACEAE}

Carpobrotus

deliciosus (L.Bolus) L.Bolus, V 305

edulis (L.) L.Bolus, V 210

Delosperma

edwardsiae L.Bolus, V 218, 221, 269, 270; V\&H 31

litorale (Kensit) L.Bolus, V 219, 550; V\&H 79

Disphyma crassifolium (L.) L.Bolus, $V 268$

Drosanthemum

brevifolium (Aiton) Schwantes, $V 222$

sp., $V \& H 63$

Lampranthus

conspicuus (Haw.) N.E.Br., V 260; V\&H 64

dependens (L.Bolus) L.Bolus, VFC 180

sociorum (L.Bolus) N.E.Br., V\&H 32

stipulaceus (L.) N.E.Br., V 230

sp., $v 220$

Conophytum bilobum (Marloth) N.E.Br., VFC 176

Ruschia tenella (Haw.) Schwantes V 565; V\&H 65

\section{MYRICACEAE}

Myrica quercifolia $L$.

MYRTACEAE

*Leptospermum laevigatum (Caertn.) F.Muell, V 264

OLEACEAE

Olea exasperata Jacq.

OXALIDACEAE

Oxalis spp., V 310,224

PLANTAGINACEAE

*Plantago lanceolata $L$., V 525

PLUMBAGINACEAE

Limonium scabrum (Thumb.) Kuntze var. scabrum. V\&H 60

POLYGALACEAE

Muraltia ericoides (Burm.f.) Steud., V 499

Polygala

fruticosa P.J.Bergius, V 295

microlopha $D C$. var. gracilis Levyns, $V 314$

myrtifolia $L ., V 346$

PRIMULACEAE

*Anagallis arvensis $L, V 291,519$

PROTEACEAE

*Hakea sericea Schrad., V 361

Protea neriifolia $R . B r ., V 237$

Leucadendron salignum P.J.Bergius, V 263

\section{RANUNCULACEAE}

Knowltonia vesicatoria (L.f.) Sims subsp. grossa H.Rasm., V 340

\section{RHAMNACEAE}

Phylica

axillaris Lam

var axillaris, VFC 174

var maritima Pillans, VFC 228

confusa Pillans, V\&H 50

purpurea Sond var. pearsonii Pillans, V 245

strigulosa Sond., V\&H 29

\section{ROSACEAE}

Cliffortia

falcata $L . f, V 265$

serpyllifolia Cham \& Schlechid. V 228, 542

sp. $V 3 / 3$
RUBIACEAE

Anthospermum

aethiopicum $L$., $V 249,5 / 2$

prostratum Sond, V 251

RUTACEAE

Agathosma

apiculata G.F.W.Mey., V 275

capensis (L.) Dümmer, V 312

ovata (Thunb.) Pillans, V 232, 337

Euchaetis burchellii Dümmer, Wi 2310

SANTALACEAE

Colpoon compressum P.J.Bergius, V 229, 342

Thesidium

fragile (Thunb.) Sond.

microcarpum (A.DC.) A.DC., V 231, 298

podocarpum (A.DC.) A.DC., V 278

Thesium

lisae-mariae Stauffer, V 330

nigromontanum Sond., V\&H 51

sertulariastrum A.W.Hill, V 226

virgatum Lam., V\&H 28

\section{SAPOTACEAE}

Sideroxylon inerme $L$. subsp. inerme, $V \& H 67$

SCROPHULARIACEAE

Graderia scabra (L.f.) Benth., Bo 8157

Phyllopodium rustii (Rolfe) Hilliard, V 284

Sutera

hispida (Thumb.) Druce, VFC $163 a$

sp., V 277, 339

\section{SELAGINACEAE}

Selago

corymbosa $L ., V$ 554, 563

dregei Rolfe, VFC 209

sp. nov., $V 234,496$

SOLANACEAE

Solanum rigescens Jacq., VFC 178

STERCULIACEAE

Hermannia

althaeifolia $L,, V 250$

angularis Jacq., V 256, 535

holosericea Jacq., V 539

lavandulifolia $L$., $V 283$

salvïifolia L.f. var. salviifolia, V 507

\section{THYMELAEACEAE}

Passerina

falcifolia C.H.Wright, V 556

vulgaris Thoday, V 498

Struthiola

ciliata (L.) Lam. subsp. angustifolia (Lam.) Peterson, V 216

parviflora Meisn., V 497

striata Lam., V 504: V\&H 73

TILIACEAE

Grewia occidentalis $L ., V 521$

VERBENACEAE

* Verbena bonariensis L., V 506

VISCACEAE

Viscum capense $L$. $f$ subsp hoolei Wiens, V 225

ZYGOPHYLLACEAE

Zygophyllum morgsana L., V 274, 369 